Cochrane Database of Systematic Reviews

\title{
Targeted interventions and approaches to care for people living with chronic obstructive pulmonary disease and at least one other long-term condition: a mixed methods review (Protocol)
}

Janjua S, McDonnell MJ, Harrison SL, Dennett EJ, Stovold E, Holland AE

Janjua S, McDonnell MJ, Harrison SL, Dennett EJ, Stovold E, Holland AE.

Targeted interventions and approaches to care for people living with chronic obstructive pulmonary disease and at least one other longterm condition: a mixed methods review.

Cochrane Database of Systematic Reviews 2019, Issue 8. Art. No.: CD013384.

DOI: 10.1002/14651858.CD013384.

www.cochranelibrary.com

Targeted interventions and approaches to care for people living with chronic obstructive pulmonary disease and at least one other long- 


\section{TABLE OF CONTENTS}

HEADER . . . . . . . . . . . . . . . . . . . . . . . . . . . . . . . . . . . . . . . 1

ABSTRACT . . . . . . . . . . . . . . . . . . . . . . . . . . . . . . . . . . . . . . . 1

BACKGROUND . . . . . . . . . . . . . . . . . . . . . . . . . . . . . . . . . . . 1

Figure 1. . . . . . . . . . . . . . . . . . . . . . . . . . . . . . . . . . . . . .

OBJECTIVES . . . . . . . . . . . . . . . . . . . . . . . . . . . . . . . . . . . . . . . . .

METHODS . . . . . . . . . . . . . . . . . . . . . . . . . . . . . . . . . . . . . . . . . 5

ACKNOWLEDGEMENTS . . . . . . . . . . . . . . . . . . . . . . . . . . . . . . . . . . . . . . . . . . .

REFERENCES . . . . . . . . . . . . . . . . . . . . . . . . . . . . . . . . . . . . . . 11

ADDITIONAL TABLES . . . . . . . . . . . . . . . . . . . . . . . . . . . . . . . . . . . . . . . . . . 14

APPENDICES . . . . . . . . . . . . . . . . . . . . . . . . . . . . . . . . . . . . . . . . 15

WHAT'S NEW . . . . . . . . . . . . . . . . . . . . . . . . . . . . . . . . . . . . . . . . . . . . 17

CONTRIBUTIONS OF AUTHORS . . . . . . . . . . . . . . . . . . . . . . . . . . . . . . . . . . . . . . .

DECLARATIONS OF INTEREST . . . . . . . . . . . . . . . . . . . . . . . . . . . . . . . . . . . . . . .

SOURCES OF SUPPORT . . . . . . . . . . . . . . . . . . . . . . . . . . . . . . . . . . . . . . . . . . . . . . .

Targeted interventions and approaches to care for people living with chronic obstructive pulmonary disease and at least one other longterm condition: a mixed methods review (Protocol)

Copyright $\odot 2019$ The Cochrane Collaboration. Published by John Wiley \& Sons, Ltd. 
[Prototype Protocol]

\title{
Targeted interventions and approaches to care for people living with chronic obstructive pulmonary disease and at least one other long-term condition: a mixed methods review
}

\author{
Sadia Janjua ${ }^{1}$, Melissa J McDonnell ${ }^{2}$, Samantha L Harrison ${ }^{3}$, Emma J Dennett ${ }^{1}$, Elizabeth Stovold ${ }^{1}$, Anne E Holland ${ }^{4,5,6}$ \\ ${ }^{1}$ Cochrane Airways, Population Health Research Institute, St George's, University of London, London, UK. ${ }^{2}$ Department of Respiratory \\ Medicine, Galway University Hospital, Galway, Ireland. ${ }^{3}$ School of Health and Social Care, Teesside University, Middlesbrough, UK. \\ ${ }^{4}$ Physiotherapy, Alfred Health, Melbourne, Australia. ${ }^{5}$ Discipline of Physiotherapy, School of Allied Health, Human Services and Sport, \\ La Trobe University, Melbourne, Australia. ${ }^{6}$ Institute for Breathing and Sleep, Melbourne, Australia
}

Contact address: Sadia Janjua, Cochrane Airways, Population Health Research Institute, St George's, University of London, London, SW17 0RE, UK. sjanjua@sgul.ac.uk.

Editorial group: Cochrane Airways Group.

Publication status and date: Edited (no change to conclusions), published in Issue 8, 2019.

Citation: Janjua S, McDonnell MJ, Harrison SL, Dennett EJ, Stovold E, Holland AE. Targeted interventions and approaches to care for people living with chronic obstructive pulmonary disease and at least one other long-term condition: a mixed methods review. Cochrane Database of Systematic Reviews 2019, Issue 8. Art. No.: CD013384. DOI: 10.1002/14651858.CD013384.

Copyright (C) 2019 The Cochrane Collaboration. Published by John Wiley \& Sons, Ltd.

\section{A B S T R A C T}

This is a protocol for a Cochrane Review (Prototype). The objectives are as follows:

1. To assess the effectiveness of integrated care pathways compared to routine care for people with COPD and one or more common comorbidities (quantitative data, RCTs).

2. To assess the effectiveness of an adapted or targeted single COPD intervention (simple or complex) that is aimed at changing the management of people with COPD and one or more common comorbidities (quantitative data, RCTs).

3. To identify emerging themes that describe the views and experiences of patients, carers and healthcare professionals when receiving or providing care to manage multi morbidities (qualitative data).

4. To use a mixed methods approach to combine quantitative and qualitative data resulting from objectives 1, 2 and 3, provided that we find relevant data. If we find that we are unable to combine quantitative data and qualitative textual themes, we will present the data and themes separately.

5. To identify any gaps in the evidence as a recommendation for further research.

\section{B A C K G R O U N D}

\section{Description of condition}

It is estimated that the global population of people aged 60 and over will triple to 2.1 billion by 2050 , with an increase of $32 \%$

Targeted interventions and approaches to care for people living with chronic obstructive pulmonary disease and at least one other longterm condition: a mixed methods review (Protocol)

Copyright $\odot 2019$ The Cochrane Collaboration. Published by John Wiley \& Sons, Ltd. 
in more developed countries, and $10 \%$ to $19 \%$ in less developed countries (United Nations 2013). As more people live longer, the number of chronic physical conditions that they may have are likely to increase (Garin 2016; Academy of Medical Sciences Report 2018).

The term 'multi morbidity' is commonly used for the co-existence of two or more chronic conditions, neither (or none) of which are considered to be an index condition (Academy of Medical Sciences Report 2018). Multimorbidity is associated with increasingly poor health outcomes (including reduced quality of life; impaired functional status; weakened physical and mental health; increased risk of re-admission to hospital; and mortality) (Barnett 2012; Holland 2016; NICE 2018).

Prevalence of multi morbidity on a global level may be difficult to determine as access to health care and diagnosis of chronic conditions vary from country to country (Academy of Medical Sciences Report 2018). However, one cross-sectional study has recently shown prevalence of multi morbidity increases from over $40 \%$ to $70 \%$ in those aged 60 to 69 years across several low, middle- and high-income countries (China, Finland, Ghana, India, Mexico, Poland, Russia, South Africa and Spain) (Garin 2016). It is estimated that approximately one in four people in the UK live with two or more long-term conditions, rising to twothirds in people aged 65 and over (Barnett 2012; NHS England 2018; Salisbury 2018). In the countries investigated by the Garin 2016 study, chronic obstructive pulmonary disease (COPD) was found to be associated with angina and asthma.

COPD is the name for a group of lung diseases (including bronchitis and emphysema). COPD occurs in adults (aged 35 years and over), and is characterised by chronic airflow obstruction that interferes with normal breathing and is not fully reversible (World Health Organisation 2018). Diagnosis of COPD is based on people experiencing day-to-day symptoms such as coughing, breathlessness (dyspnoea), and wheezing and frequent chest infections. People may also experience periodic exacerbations (flare-ups). The risk factors for COPD include smoking and environmental exposures leading to abnormalities of the airways and alveoli (World Health Organisation 2018; GOLD 2019). Further tests to diagnose COPD include spirometry, in which "the volume of air forcibly exhaled from the point of maximal inspiration (forced vital capacity, FVC), and the volume of air exhaled during the

first second (forced expiratory volume in one second, FEVı )" are

measured. A FEVı /FVC ratio of less than 0.70 is an indicator for airway obstruction (GOLD 2019).

As COPD is associated with a high prevalence of multi morbidities (Smith 2014), it is common for people with COPD to have more than one co-existing long-term health condition that can vary in nature and severity (Cavailles 2013; Holland 2016). People with more severe COPD (GOLD stage D) are likely to have a higher number of co-morbidities (Raherison 2018), which puts them at a higher risk of mortality compared to people with mild or moderate
COPD, or those without COPD and co-existing long-term health conditions (Mannino 2008; Divo 2012; Hanlon 2018).

Common long-term conditions that co-exist with COPD are cardiovascular disease, hypertension, diabetes, asthma, and lung cancer (Hillas 2015). People may also live with long-term condition system complexes such as frailty and chronic pain (Holland 2016; Andenæs 2018). In this review we will treat pain as an outcome, rather than a condition. These long-term conditions may or may not be related to COPD.

In this review, we will focus on people with COPD living with one or more long-term physical conditions (also referred to as comorbidities of COPD) (Holland 2016; Smith 2016). We do not plan to include people with conditions caused by COPD treatments, such as pneumonia, or ongoing conditions such as learning disability, sensory impairment such as sight or hearing loss, and alcohol and substance misuse.

\section{Description of interventions}

Interventions (treatments) for people with COPD are either aimed at helping them to manage the symptoms of COPD in day-today life, or are treatment of exacerbations (flare-ups). For treating the symptoms, there are drugs including inhaled therapies (such as

long-acting beta ${ }^{2}$-agonists, long-acting muscarinic antagonists, and inhaled corticosteroids), phosphodiesterases and antibiotics, as well as physical interventions such as pulmonary rehabilitation, physical therapy (e.g. exercise), ventilation (e.g. non-invasive ventilation (NIV)). For treating exacerbations there are inhaled therapies, antibiotics and ventilation.

In this review we plan to look at COPD interventions which target the co-morbidity (e.g. pulmonary rehabilitation where the exercise component is taken in the swimming pool to enable people with physical conditions to take part (McNamara 2013)). We will also include interventions to improve the overall management of people with COPD and one or more chronic conditions.

We have created a framework from the GOLD 2019 guidelines and the Cochrane Airways subtopic list, from which we intend to create an evidence (gap) map and use it as a basis for the analysis (Table 1).

\section{How will the intervention work?}

Long-term conditions other than COPD may interfere with the delivery of the COPD intervention. An example of people with multi morbidity engaging with an intervention differently from those with COPD alone is seen in pulmonary rehabilitation, one of the more effective treatments for people with COPD (McCarthy 2015). Researchers have shown that people with multimorbid COPD are more likely than people with COPD only to either decline to enrol for treatment or, once enrolled, to drop out of the programme or not attend sessions regularly (Fischer 2009; 
Keating 2011; Hayton 2013). Patients are more likely to drop out of pulmonary rehabilitation programmes as a result of symptoms of other co-morbidities (Blackstock 2018). Furthermore, researchers have evaluated the impact of co-existing conditions on outcomes of a pulmonary rehabilitation intervention for people with COPD which showed that depending on the co-existing condition, pulmonary rehabilitation outcomes can be positive or negative (Crisafulli 2008; Carreiro 2013; Walsh 2013; Holland 2016). Targeted interventions can help people take part in pulmonary rehabilitation programmes: a targeted water-based exercise component of a pulmonary rehabilitation programme was shown to be more effective than land-based exercise (McNamara 2013). We therefore intend to summarise evidence from randomised controlled trials (RCTs) which target a COPD intervention to take account of another co-morbidity.

People with multi morbidities may be taking multiple drugs for each individual condition; for example, prescribed drugs, overthe-counter treatments, herbal remedies or dietary supplements. This is called polypharmacy and $16.4 \%$ of people over the age of 65 years are estimated to be taking 10 or more treatments each day (Guthrie 2012; Duerden 2013). This can lead to unfavourable drug interactions and practical issues with remembering to take so many medications in a day. We will include interventions which help people adapt to taking multiple medications; we will not, however, be looking at polypharmacy interventions which aim to optimise a patient's drugs and reduce harmful drug interactions.

People with multi morbidities may also have to see many differ- ent healthcare professionals to help them with various different elements of their different long-term conditions. We will include trials which aim to streamline (or simplify) this care in some way to make it easier or better for the patient. These might include, for example, putting a patient under the care of one particular consultant who works across several hospital departments, thereby providing a holistic package of care. It may include a hospital putting together an integrated disease management programme - a map of a patient's journey for managing their condition in a particular location. We will also consider simpler interventions such as running COPD and cardiovascular clinics on the same day to reduce the number of attendances at hospital.

Anxiety and depression are common in people with COPD. Pharmacological and psychological interventions aimed at treating the anxiety and depression are explored in a suite of Cochrane Reviews (Pollok 2018; Pollok 2019; Usmani 2011; Usmani 2017). Because the interventions are treating the co-morbidity rather than the COPD, we will not include them in this review. We will also not include studies of people with COPD who have symptoms of depression or anxiety as the sole co-morbidity.

We present a draft logic model to help us and readers to think about the interplay between different interventions and different co-morbidities (Figure 1). We intend to take this model to our Cochrane Airways Patient Advisory Group and Programme Grant Steering Group for their consideration, and also to review it in light of the evidence found in the review.

Figure I. Map to show the complexity of interventions that may be required by people with COPD depending on the number and combination of co-existing conditions they may have and the impact on short term and long term outcomes

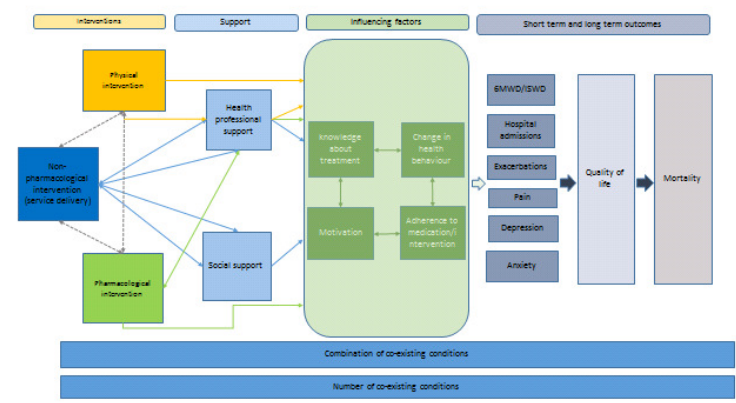

Targeted interventions and approaches to care for people living with chronic obstructive pulmonary disease and at least one other long- 


\section{Why is it important to do this review?}

Most clinical trials are designed to involve people with one condition, and exclude people with multi morbidities - which may be well over half of people who live with COPD. Therefore systematic reviews, including Cochrane Reviews, have also traditionally focused on these patients, rather than including a sample representing the true population. This means that most studies may not be applicable to people with more than one chronic condition: for example, trials may only enrol people with COPD and exclude people with asthma or heart disease. This means that we cannot be confident about applying the results of the trial to people with COPD and asthma, or people with COPD and heart disease.

There is a substantial health burden for people living with COPD and multi morbidities, with associated cost implications due to an increased need of hospital utilisation compared to patients who only have one condition (Chen 2017). People living with multi morbidity may also have to manage several symptoms, adhere to multiple drug regimens and various lifestyle recommendations, all while attending appointments with different healthcare providers (Smith 2016). Healthcare services experience higher demands as patients with multi morbidities require more frequent complex care (Barnett 2012; Rijken 2018), and these services can be fragmented (Smith 2016).

Policy makers are increasingly aware that overall care for people with multi morbidities needs to be patient-centred (i.e. care that takes a patient's needs into account, either via individual preference, or by involving the patient in making decisions about their care) and integrated (i.e. organisations and staff working together to provide seamless care through processes that are flexible and continuous) (Rijken 2016).

In addition, guidance for managing multi morbidities is limited because of the exclusion from clinical trials of people living with multi morbidity. The systematic failure of clinical trials to include people living with multi morbidity leads to care strategies that may not be suitable or helpful for the majority of people with COPD (Wyatt 2014). For example, multiple prescriptions (polypharmacy) can lead to potential interactions between conditions and medication resulting in inadequate and complex choices of treatment in terms of benefit and harm (Sinnot 2013; Muth 2018), or fragmented and poorly coordinated care packages can lead to complications such as over-hospitalisation when managing patients with multi morbidities (Sinnot 2013; Rijken 2016).

We have decided to undertake this review because the Cochrane Airways Patient Advisory Group and Programme Grant Steering Group considered this to be an important topic to be reviewed for a programme of Cochrane Airways reviews funded by the $\mathrm{Na}$ tional Institute for Health Research. The patients and healthcare professionals agreed that the systematic review should report information about the clinical effectiveness of interventions, and the views and experiences of those involved in managing multi morbidities and COPD, and identify gaps in the evidence. The review will address issues that are important for people with COPD who have co-existing conditions, as well as for healthcare providers and policy-makers.

Our scoping search of quantitative and qualitative evidence in this topic area showed there are potential studies of either quantitative, qualitative or mixed methods that investigate clinical effectiveness of interventions and patient, healthcare professional or career perspectives of interventions.

In comparison with a previous Cochrane Review (Smith 2016), we have decided to take a mixed methods approach to evaluate the evidence that exists for people living with COPD and at least one other chronic condition in this review because of concerns that interventions begun with the best intentions may not always be helpful for patients. This means we will identify studies conducted in a community or hospital setting to combine both quantitative data (numerical data from clinical trials), and qualitative data (non-numerical data from, for example, semi-structured interviews, focus group discussions and patient, career or health professional observations). To illustrate: we are aware of a local example where people with COPD and heart disease have been put under a coordinated care regime, but the patients have said they prefer separate appointments because they are shorter and they like having a reason to get out of the house.

We have deliberately left the types of intervention very broad (compared to Smith 2016), to reflect the reality of people living with COPD and other long-term health conditions in trying to make sense of a sparse literature, who nonetheless need to make decisions about how to manage their own symptoms and daily life. The interventions are aimed to address COPD rather than the full extent of multi morbidity.

There is a risk that we will not be able to meta-analyse any of the data in the review according to our analysis plan below, in which case we will map what is available and use this review to call for a reconsideration of what trials need to be funded and run.

\section{O B J E C T I VES}

1. To assess the effectiveness of integrated care pathways compared to routine care for people with COPD and one or more common comorbidities (quantitative data, RCTs). 
2. To assess the effectiveness of an adapted or targeted single COPD intervention (simple or complex) that is aimed at changing the management of people with COPD and one or more common comorbidities (quantitative data, RCTs).

3. To identify emerging themes that describe the views and experiences of patients, carers and healthcare professionals when receiving or providing care to manage multi morbidities (qualitative data).

4. To use a mixed methods approach to combine quantitative and qualitative data resulting from objectives 1, 2 and 3, provided that we find relevant data. If we find that we are unable to combine quantitative data and qualitative textual themes, we will present the data and themes separately.

5. To identify any gaps in the evidence as a recommendation for further research.

\section{MET HODS}

\section{Types of studies}

We will include the following study designs to address the objectives of this review.

- Randomised controlled trials (RCTs) to assess effectiveness of interventions (quantitative data).

- Studies based on, for example, in-depth interviews, semistructured interviews, focus group discussion, observations, and surveys to identify views, opinions and experiences of people with COPD and multi morbidity, their carers and health professionals involved in provision of care (qualitative data).

- Mixed methods studies (RCTs that also include a qualitative analysis as part of their investigations).

\section{Types of participants}

We will include adults with a primary diagnosis of COPD of any severity (e.g. Global Initiative for Chronic Obstructive Lung Disease (GOLD), or American Thoracic Society (ATS) criteria), as defined by objective evidence of airflow limitation on spirometry in the context of a significant (more than 10 pack-years) smoking history and living with at least one other long-term condition (e.g. asthma, coronary heart disease, diabetes, atrial fibrillation, heart failure, hypertension, stroke/transient Ischaemic attack, lung cancer or osteoporosis (Barnett 2012)).

We will include people with COPD who also have anxiety or depression or both, but this should not be the only co-morbidity. We will include carers and healthcare professionals when receiving or providing care to manage multi morbidities.

\section{Types of interventions}

We will include the following interventions for quantitative studies.

- An intervention aimed at changing the management of people with COPD and one (or more) co-existing long-term condition(s), which could be simple (e.g. scheduling COPD and heart clinics on the same day) or more complex (e.g. developing an integrated care package for management of people with COPD in a particular hospital and providing training to staff to deliver it), compared to routine care (or usual care, control, or no intervention). We envisage these interventions being broken into subgroups as follows.

- Organisation-wide interventions (such as introducing a new care pathway)

- Simple changes within the organisation (such as scheduling relevant clinics on the same day)

- Interventions across a wider area (such as integration between GP, hospital and pharmacy)

- Any single intervention for COPD delivered to people with COPD adapted or targeting their comorbidity (or comorbidities) (e.g. participants receiving a pulmonary rehabilitation programme tailored to take account of their comorbidities by delivering the exercise component in water rather than out of water (McNamara 2013)) compared to any other intervention. We envisage these interventions being broken into subgroups as follows.
- Pulmonary rehabilitation
- Exercise or other physical therapy
- Ventilation
- Pharmacotherapy (e.g. change of inhaler)

We will include interventions delivered in primary (community) or secondary (hospital) care.

We will exclude studies of interventions that target the comorbidity (e.g. we will exclude an intervention aimed at CVD).

We will exclude studies of pharmacological or psychological interventions that target anxiety or depression or both rather than COPD (previous Cochrane Reviews have, for example, included COPD patients with either anxiety (Usmani 2017), depression (Pollok 2018; Pollok 2019), or both anxiety and depression (Usmani 2011)).

We will exclude interventions that are designed to reduce the number of prescribed medicines or interactions between them (polypharmacy), but we will include interventions that aim to help people to manage polypharmacy.

We will exclude interventions delivered to healthcare professionals. We will include qualitative studies that explore the experiences of people taking part in the above interventions. 


\section{Types of outcome measures}

We will include the following outcomes for quantitative analysis.

\section{Primary outcomes}

- Quality of life (e.g. St. George's Respiratory Questionnaire (SGRQ), COPD assessment test (CAT))

- Exacerbations (as defined by trialists, depending on the data available, we will extract either number of participants experiencing one or more exacerbation, or the exacerbation rate, or both)

\section{Secondary outcomes}

- Functional status (6-minute walk distance (6MWD)/ incremental shuttle walk test (ISWD))

- All-cause hospital admissions (also as a proxy for use of services, e.g. reduction of use)

- Respiratory hospital admissions

- Mortality (all causes)

- Pain (as reported in trials)

- Anxiety symptoms (measured by e.g. Hospital Anxiety and Depression Scale (HADS))

- Depression symptoms (measured by e.g. HADS)

For qualitative synthesis, we will identify themes that arise from the data; however, we expect to find the following themes.

- Experience of living and treating more than one condition.

- Experience of services' or healthcare barriers or facilitators to intervention participation

- Intervention delivery

- Impact on quality of life and mood

- Mode of delivery

We will include studies regardless of whether or not they report our pre-defined outcomes.

We will include studies of short-term and long-term duration of interventions.

\section{Search methods for identification of studies}

\section{Electronic searches}

We will search for studies in the following databases and trials registries.

- Cochrane Airways Register of Trials through the CRS, from inception to date;

- Cochrane Central Register of Controlled Trials

(CENTRAL, in the Cochrane Library) through the Cochrane Register of Studies (CRS), from inception to date;

- MEDLINE Ovid SP 1946 to date;
- Embase Ovid 1974 to date;

- PsycINFO Ovid Sp 1974 to date;

- CINAHL EBSCO (Cumulative Index to Nursing and Allied Health Literature) 1937 to date;

- Web of Science Core Collection 1970 to date;

- US National Institutes of Health Ongoing Trials Register ClinicalTrials.gov;

- World Health Organization International Clinical Trials Registry Platform ( ICTRP).

Searches for qualitative and quantitative studies will be run separately using appropriate study design filters. We will combine search terms for the target population with the Cochrane Highly Sensitive Search Strategy to identify reports of RCTs (Lefebvre 2009), and terms from the search strategies developed and tested by DeJean 2016 to find reports of qualitative studies. We list the proposed MEDLINE search strategies in Appendix 2; these were developed by ES, with input from the other authors, and were peer-reviewed by another Information Specialist using the PRESS checklist (McGowan 2016).

The MEDLINE search strategies will be adapted appropriately for use in the other databases. We will search all databases from their inception to the present, and there will be no restriction on language of publication. We will not limit the search strategy with respect to population characteristics such as age, gender or race.

\section{Searching other resources}

We will check the reference lists of all primary studies and review articles for additional references. We will use the Epistemonikos database to search for relevant systematic reviews ( www.epistemonikos.org). We will search relevant manufacturers' websites for study information.

We will search for errata or retractions from included studies published in full text on PubMed and report the date this was done within the review.

\section{Data collection and analysis}

\section{Selection of studies}

We anticipate retrieving a large number of search results, and therefore we are planning to use Cochrane's 'Screen4Me' workflow to help assess the results of our search for RCTs. Screen4Me comprises three components: known assessments - a service that matches records in the search results to records that have already been screened in Cochrane Crowd and have been labelled as 'RCT' or as 'Not an RCT'; the RCT classifier - a machine-learning model that distinguishes RCTs from non-RCTs (Marshall 2018); and if 
appropriate, Cochrane Crowd - Cochrane's citizen science platform where 'the crowd' help to identify and describe health evidence (Noel-Storr 2018).

Following this, two of three review authors (SJ, ES and ED) will screen each title and abstract of the remaining search results independently and code them as 'retrieve' (eligible or potentially eligible/unclear) or 'do not retrieve'. We will retrieve the full-text study reports of all potentially eligible studies and two of three review authors (SJ, ES and ED) will independently screen each full text for inclusion, recording the reasons for exclusion of ineligible studies.

We will resolve any disagreement through discussion or, if required, we will consult a third review author $(\mathrm{SH})$. We will identify and exclude duplicates and collate multiple reports of the same study so that each study, rather than each report, is the unit of interest in the review. We will record the selection process in sufficient detail to complete a PRISMA flow diagram and 'Characteristics of excluded studies' table (Moher 2009).

\section{Data extraction and management}

Two authors (SJ, ED, ES) will screen citations in Rayyan (Mourad 2016). One review author (SJ) will pilot the data extraction form on at least one quantitative, one qualitative and one mixed methods study before extracting data from the rest of the included studies. We will extract data into Microsoft Excel.

\section{Quantitative studies}

One review author (SJ) will extract the following study characteristics from included studies.

- Methods: study design, total duration of study, details of any 'run-in' period, number of study centres and location, study setting, withdrawals and date of study.

- Participants: N, mean age, age range, gender, severity of condition, diagnostic criteria, baseline lung function, smoking history, inclusion criteria and exclusion criteria.

- Interventions: intervention, comparison, concomitant medications and excluded medications.

- Outcomes: primary and secondary outcomes specified and collected (e.g. confidence intervals, P values, measurement scales used), and time points reported. Definitions used to diagnose an exacerbation will be sought and recorded.

- Notes: funding for studies and notable conflicts of interest of trial authors.

A second review author (ED) will spot-check $10 \%$ to $20 \%$ of characteristics extracted from studies by the first author.

Two review authors (SJ and ED) will independently extract outcome data from included studies. We will note in the 'Characteristics of included studies' table if outcome data were not reported in a usable way. We will resolve disagreements by consensus or by involving a third person/review author $(\mathrm{SH})$. One review author (SJ) will transfer quantitative data into the Review Manager 5 (RevMan 5) file (Review Manager 2014). We will double-check that data are entered correctly by comparing the data presented in the systematic review with the study reports. A second review author (ED) will spot-check study characteristics for accuracy against the study report.

\section{Qualitative studies}

In order to capture context, two review authors (SJ and ED) will extract the following study characteristics from included studies.

- Study details: country, study type (e.g. focus group, semistructured interviews, structured interviews, surveys), dates, source of funding, objectives

- Participants: N, mean age, age range, gender, severity of condition, diagnostic criteria, baseline lung function, smoking history, inclusion criteria and exclusion criteria

- Methods: sampling, setting (e.g. community or hospital), data collection (e.g. how the authors conducted the study, length of interviews, whether interviews were recorded, use of interview guide, data collected until achievement of thematic saturation), data analysis (e.g. method of analysis of transcripts, framework used, coding, thematic map)

- Results: authors' interpretations, quotes from participants provided by authors.

\section{Assessment of risk of bias}

\section{Quantitative studies}

Two review authors (SJ, and ED) will assess risk of bias independently for each study using the criteria outlined in the Cochrane Handbook for Systematic Reviews of Interventions (Higgins 2011). We will resolve any disagreements by discussion or by involving another author $(\mathrm{SH})$. We will assess the risk of bias according to the following domains.

- Random sequence generation

- Allocation concealment

- Blinding of participants and personnel

- Blinding of outcome assessment

- Incomplete outcome data

- Selective outcome reporting

- Other bias

We will judge each potential source of bias as high, low or unclear and provide a quote from the study report together with a justification for our judgement in the 'Risk of bias' table. We will summarise the risk of bias judgements across different studies for each of the domains listed. We will consider blinding separately for different key outcomes where necessary (e.g. for unblinded outcome 
assessment, risk of bias for all-cause mortality may be very different than for a patient-reported quality of life scale). Where information on risk of bias relates to unpublished data or correspondence with a trialist, we will note this in the 'Risk of bias' table. When considering treatment effects, we will take into account the risk of bias for the studies that contribute to that outcome.

We will present a 'Risk of bias' table for all studies.

\section{Qualitative studies}

Two review authors (SJ and ED) will assess risk of bias independently for each study using the criteria outlined by the Cochrane Quality and Intervention Methods Group (Hannes 2011). We will resolve any disagreements by discussion or by involving another author $(\mathrm{SH})$. We will assess the risk of bias according to the following criteria.

- Quality of reporting (explicitness in reporting of all study aspects)

- Methodological rigour (validity and reliability of study design and process)

- Conceptual depth and breadth (are reported aims, rationale or theory reflected in the study design, process and findings?)

We will use the Critical Appraisal Skills programme (CASP) checklist to assess risk of bias. We will present risk of bias of studies in tabular format.

We will assess the risk of bias after the identification of relevant data when making judgements about relative strength of messages in the included research.

\section{Mixed methods studies}

We will use the Mixed Methods Appraisal Tool (MMAT) to assess risk of bias (Pluye 2011; Hong 2018). Two review authors (SJ and ED) will assess risk of bias independently for each study using the criteria outlined by the MMAT. We will resolve any disagreements by discussion or by involving another author $(\mathrm{SH})$. We will assess the risk of bias according to the following criteria.

- Is there an adequate rationale for using a mixed methods design to address the research question?

- Are the different components of the study effectively integrated to answer the research question?

- Are the outputs of the integration of qualitative and quantitative components adequately addressed?

- Are divergences and inconsistencies between quantitative and qualitative results adequately addressed?

- Do the different components of the study adhere to the quality criteria of each tradition of the methods involved?

\section{Measurement of treatment effect}

\section{Quantitative treatment effects}

We will analyse dichotomous data as odds ratios (OR) and continuous data as the mean difference (MD) or standardised mean difference (SMD). If data from rating scales are combined in a meta-analysis, we will ensure they are entered with a consistent direction of effect (e.g. lower scores always indicate improvement). We will undertake meta-analyses only where this is meaningful: that is, if the treatments, participants and the underlying clinical question are similar enough for pooling to make sense. If there is substantial statistical or clinical heterogeneity we will present data on graphs, but will not pool them. We will describe skewed data narratively (for example, as medians and interquartile ranges for each group).

Where multiple trial arms are reported in a single study, we will include only the relevant arms. If two comparisons (e.g. intervention A versus intervention B) are combined in the same meta-analysis, we will either combine the active arms or halve the control group to avoid double-counting. If adjusted analyses are available (ANOVA or ANCOVA), we will use these as a preference in our meta-analyses. If both change from baseline and endpoint scores are available for continuous data, we will use change from baseline unless there is low correlation between measurements in individuals. If a study reports outcomes at multiple time points, we will use endpoint data. We will use intention-to-treat (ITT) or 'full analysis set' analyses where they are reported (i.e. those where data have been imputed for participants who were randomly assigned but did not complete the study) instead of completer or per protocol analyses.

\section{Unit of analysis issues}

\section{Quantitative analysis}

For dichotomous outcomes, we will use participants, rather than events, as the unit of analysis. If rate ratios are reported in a study, we will analyse them on this basis. We will only meta-analyse data from cluster-RCTs if the available data have been adjusted (or can be adjusted), to account for the clustering.

\section{Dealing with missing data}

We will contact investigators or study sponsors in order to verify key study characteristics and obtain missing numerical outcome data where possible (e.g. when a study is identified as an abstract only). Where this is not possible, and the missing data are thought to introduce serious bias, we will take this into consideration in the GRADE rating for affected outcomes. 


\section{Assessment of certainty of evidence}

\section{Quantitative data}

We will use the five GRADE considerations (risk of bias, consistency of effect, imprecision, indirectness, and publication bias) to assess the quality of the body of evidence as it relates to the studies that contribute data for the prespecified outcomes. We will use the methods and recommendations described in Section 8.5 and Chapter 12 of the Cochrane Handbook for Systematic Reviews of Interventions ( Higgins 2011), using GRADEpro GDT software (GRADEpro GDT; Guyatt 2008). We will justify all decisions to downgrade the quality of studies in the footnotes of the table, and we will make comments to aid the reader's understanding of the review where necessary. We will present GRADE findings in a 'Summary of findings' table.

\section{Qualitative data}

We will use the GRADE Confidence in the Evidence from Reviews of Qualitative Research (CERQual) approach to assess our confidence in the evidence of effectiveness arising from studies evaluating interventions (Lewin 2015). This assessment will allow us to make judgements on the following four domains.

- Methodological limitations of included studies

- Relevance of contributing studies to the research question

- Coherence of study findings

- Adequacy of data supporting the study findings

We will use the methods and recommendations described in chapter 20 of the Cochrane Handbook for Systematic Reviews of Interventions ( Higgins 2011). We will summarise findings of the four domains for each outcome in a CERQual Qualitative Evidence Profile (Lewin 2015). We will rate the overall assessment of confidence of evidence as high, moderate, low or very low. We will present CERQual findings in a 'Summary of findings' table. We will justify all decisions to downgrade the quality of studies in the footnotes of the table, and we will make comments to aid the reader's understanding of the review where necessary.

\section{Assessment of heterogeneity}

\section{Quantitative data}

We will use the $\mathrm{I}^{2}$ statistic to measure heterogeneity among the studies in each analysis where possible. We will conduct a metaanalysis using a random-effects model as we anticipate that the interventions are likely to be varied. As we are uncertain of the intervention effects, we will explore possible causes of heterogeneity if there are sufficient studies.
We will consider the following $\mathrm{I}^{2}$ ranges in the analyses (Higgins 2011).

1. $0 \%$ to $40 \%$ : might not be important

2. $30 \%$ to $60 \%$ : may represent moderate heterogeneity

3. $50 \%$ to $90 \%$ : may represent substantial heterogeneity

4. $75 \%$ to $100 \%$ : considerable heterogeneity

If we find that there is substantial statistical heterogeneity, we will check the data for accuracy. If unexplained substantial heterogeneity persists, we will consider whether it is more appropriate to present the standardised effect of each study in a forest plot without performing a meta-analysis.

\section{Assessment of reporting bias}

\section{Quantitative data}

If we are able to pool more than 10 studies, we will create and examine a funnel plot to explore possible small-study and publication biases.

\section{Data synthesis}

\section{Quantitative data}

We will use RevMan 5 to perform quantitative data syntheses (meta-analyses) (Review Manager 2014); and where data for population or interventions are similar, we will pool such data. If it is not possible to pool data, we will present the data in forest plots to show the range of effect sizes where possible or tabulate and describe in the text. If we do find that there is heterogeneity within the studies we identify, we will take measures to ensure that we limit variation by calculating standardised effect sizes, grouping studies according to interventions and outcomes, and use the random-effects model in the analyses (Ioannidis 2008). For studies in which it is not possible to perform statistical analysis, we will consider presenting data graphically and narratively using recommendations in the latest version of the Cochrane Handbook for Systematic Reviews of Interventions (in press).

\section{Qualitative data}

Where studies are similar in design we will synthesise data using a thematic analysis (Thomas 2008). This method allows us to identify important or recurrent themes that arise from studies and summarise them under thematic headings. We will tabulate information, allowing identification of prominent themes and offering structured ways of dealing with the data in each theme. This type of synthesis will help us to identify emerging themes that describe the experiences of patients, carers and healthcare professionals when receiving or providing care to manage multi morbidities. 
We will initially analyse career and healthcare professional data separately to identify, for example, conflicting views or experiences. If we find that the views and experiences are similar, we will combine the two subgroups in subsequent syntheses.

We will identify themes that describe the views and experiences of carers and healthcare professionals when receiving or providing care to manage multi morbidities. We will initially analyse career and healthcare professional data separately to identify, for example, conflicting views or experiences. If we find that the views and experiences are similar, we will combine the two subgroups in subsequent analyses.

\section{Combining quantitative and qualitative data}

We will use the methods and recommendations described in chapter 20 of the Cochrane Handbook for Systematic Reviews of Interventions, and methods outlined by the Cochrane Qualitative and Implementation Methods Group ( Higgins 2011 and Harden 2018, respectively). There are two main approaches to integrating qualitative and quantitative data: sequential and convergent. The sequential approach involves synthesising qualitative and quantitative analyses separately, followed by using common frameworks to integrate the findings across syntheses. We will use the sequential approach to integrate qualitative data to explain quantitative findings. We will analyse quantitative data at the first stage, followed by synthesis of qualitative data in the second stage.

Integrating the qualitative syntheses with the quantitative analyses can be achieved by using a 'matrix' to compare and contrast findings across both types of evidence. The matrix will help to identify gaps in the evidence. This approach can help us to understand why heterogeneity that we may find in the quantitative analyses exists. Other approaches include the development of a 'logic model' by providing a common framework within which both quantitative and qualitative evidence can contribute (Harden 2018). We acknowledge that the methods for integration are dependent on the quantity of studies and extracted evidence available, and quality of description within included studies (e.g. intervention content, context, and study findings). For divergent data (qualitative data that does not match the quantitative data), we will aim to resolve divergence (deviation of the qualitative data from the quantitative data, or vice versa (Erzberger 1997)) where possible by providing a narrative explanation according to research and knowledge of the topic area.

Combining both quantitative and qualitative data will provide better understanding of why some interventions are successful and why other interventions are not so successful. This approach will help to add richness and depth to quantitative findings which is not methodologically possible to do when interpreting quantitative data alone. We can identify areas where quantitative research may be lacking but appears to be important to patients, carers or health professionals (as identified from qualitative research).

\section{Subgroup and investigation of heterogeneity}

We will investigate heterogeneity such as statistical heterogeneity of quantitative data. We plan to perform subgroup analyses if we find heterogeneity using the following pre-specified groups.

- Number of multi morbidities in addition to COPD ( $\leq 2$ conditions versus $\geq 3$ conditions)

- Duration of intervention ( $<3$ months versus $\geq 3$ months)

We will use the following outcomes in the subgroup analyses.

- Quality of life

- Functional status

- Hospital admissions

- Exacerbations

We will use the formal test for subgroup interactions in Review Manager 5 (Review Manager 2014)

\section{Sensitivity analysis}

We plan to carry out sensitivity analysis excluding studies in which one or more risk of bias domains is judged to be high risk of bias.

\section{Assessment of bias conducting the systematic review}

We will conduct the review according to this published protocol and justify any deviations from it in the 'Differences between protocol and review' section of the systematic review.

\section{ACKNOWLEDGEMENTS}

Sally Spencer was the Editor for this protocol and commented critically on the protocol.

With thanks to the editorial team for their contributions listed below, and to Doug Salzwedel for peer reviewing the search strategy. Thank you to the patient advisory group and the steering group for the programme grant for continued advice and support.

The authors and Airways editorial team are grateful to the following peer reviewers for their time and comments:

- Audrey Tan, Network Support Fellow, Cochrane Circulation and Breathing Network;

- Prof Susan M Smith, Department of General Practice, RCSI, Dublin, Ireland;

- Robin Carr, retired GP, Oxfordshire; and

- Anke Lenferink PhD, Department of Health Technology and Services Research, Faculty of Behavioural, Management and

Targeted interventions and approaches to care for people living with chronic obstructive pulmonary disease and at least one other long- 10 term condition: a mixed methods review (Protocol)

Copyright (๑) 2019 The Cochrane Collaboration. Published by John Wiley \& Sons, Ltd. 
Social sciences, Technical Medical Centre, University of Twente,

Enschede, the Netherlands.

The Background and Methods sections of this protocol are based on a standard template used by Cochrane Airways.

\section{R E F E R E N C E S}

\section{Additional references}

\section{Academy of Medical Sciences Report 2018}

Academy of Medical Sciences. Multimorbidity: a priority for global health research. acmedsci.ac.uk/file-download/ 82222577 [accessed May 2019] 2018:1-127.

\section{Andenæs 2018}

Andenæs R, Momyr A, Brekke I. Reporting of pain by people with chronic obstructive pulmonary disease (COPD): comparative results from the HUNT3 population-based survey. BMC Public Health 2018;18:181. DOI: 10.1186/ s12889-018-5094-5

\section{Barnett 2012}

Barnett K, Mercer SW, Norbury M, Watt G, Wyke S, Guthrie B. Epidemiology of multi morbidity and implications for health care, research, and medical education: a cross-sectional study. Lancet 2012;380(9836): 37043. DOI: $10.1016 / S 0140-6736(12) 60240-2$

Blackstock 2018

Blackstock FC, ZuWallack R, Nici L, Lareau SC. Why don't our patients with chronic obstructive pulmonary disease listen to us? The enigma of non adherence. Annals of the American Thoracic Society 2016;13(3):317-23.

\section{Carreiro 2013}

Carreiro A, Santos J, Rodrigues F. Impact of co morbidities in pulmonary rehabilitation outcomes in patients with chronic obstructive pulmonary disease. Revista Portuguesa de Pneumologia 2013;19:106-13.

\section{Cavailles 2013}

Cavailles A, Brinchault-Rabin G, Dixmier A, Goupil F, Gut-Gobert C, Marchand-Adam S, et al. Comorbidities of COPD. European Respiratory Review 2013;22:454-75.

\section{Chen 2017}

Chen W, FitzGerald JM, Sin DD, Sadatsafavi M. Excess economic burden of co morbidities in COPD: a 15-year population-based study. European Respiratory Journal 2017; 50:1700393.

\section{Crisafulli 2008}

Crisafulli E, Costi S, Luppi F, Cirelli C, Coletti C, Fabbri $\mathrm{LM}$, et al. Role of co morbidities in a cohort of patients with COPD undergoing pulmonary rehabilitation. Thorax 2008;63:487-92.

\section{DeJean 2016}

Dejean D, Giacomini M, Simeonov D, Smith A. Finding qualitative research evidence for health technology assessment. Qualitative Health Research 2016;26(10): 1307-17. DOI: $10.1177 / 1049732316644429$

Divo 2012

Divo M, Cote C, de Torres JP, Casanova C, Martin JM, Pinto-Plata V, et al. Comorbidites and risk of mortality in patients with chronic obstructive pulmonary disease. American Journal of Respiratory and Critical Care Medicine 2012;186(2):155-61.

\section{Duerden 2013}

Duerden M, Avery T, Payne R (The King's Fund). Polypharmacy and medicines optimisation: making it safe and sound. www.kingsfund.org.uk/sites/default/files/ field/field_publication_file/polypharmacy-and-medicinesoptimisation-kingsfund-nov13.pdf 2013.

Erzberger 1997

Erzberger C, Prein G. Triangulation: validity and empirically-based hypothesis construction. Quality \& Quantity 1997;31(2):141-54

\section{Fischer 2009}

Fischer MJ, Scharloo M, Abbink JJ, van 't Hul AJ, van Ranst D, Rudolphus A, et al. Drop-out and attendance in pulmonary rehabilitation: the role of clinical and psychosocial variables. Respiratory Medicine 2009;103(10): 1564-71.

\section{Garin 2016}

Garin N, Koyanagi A, Chatterji S, Tyrovolas S, Olaya B, Leonardi M, et al. Global multi morbidity patterns: a crosssectional, population-based, multi-country study. Journals of Gerontology. Series A, Biological Sciences and Medical Sciences 2016;71(2):205-14.

\section{GOLD 2019}

Global initiative for chronic obstructive lung disease. Global strategy for the diagnosis, management, and prevention of chronic obstructive pulmonary disease (2019 report). goldcopd.org/wp-content/uploads/2018/11/GOLD-2019v1.7-FINAL-14Nov2018-WMS.pdf (accessed prior to 22 January 2019).

\section{GRADEpro GDT [Computer program]}

McMaster University (developed by Evidence Prime ). GRADEpro GDT. Version accessed prior to 22 January 2019. Hamilton (ON): McMaster University (developed by Evidence Prime ), 2015.

\section{Guthrie 2012}

Guthrie B, Makubate B. The rising tide of polypharmacy and potentially serious drug interactions 1995-2010: repeated cross-sectional analysis of dispensed prescribing in

Targeted interventions and approaches to care for people living with chronic obstructive pulmonary disease and at least one other long- I I term condition: a mixed methods review (Protocol)

Copyright $\odot 2019$ The Cochrane Collaboration. Published by John Wiley \& Sons, Ltd. 
one region. Primary Health Care Research \& Development 2012;13 (supplement 1)(45):2E2.

\section{Guyatt 2008}

Guyatt GH, Oxman AD, Vist GE, Kunz R, FalckYtter Y, Alonso-Coello P, et al. GRADE: an emerging consensus on rating quality of evidence and strength of recommendations. BMJ 2008;336:924. DOI: 10.1136/ bmj.39489.470347.AD

\section{Hanlon 2018}

Hanlon P, Nicholl BI, Jani BD, McQueenie R, Lee D, Gallacher KI, et al. Examining patterns of multi morbidity, polypharmacy and risk of adverse drug reactions in chronic obstructive pulmonary disease: a cross-sectional UK Biobank study. BMJ Open 2018;8:e018404. DOI: 10.1136/bmjopen-2017-01

\section{Hannes 2011}

Hannes K. Chapter 4: Critical appraisal of qualitative research. In: Noyes J, Booth A, Hannes K, Harden A, Harris J, Lewin S, Lockwood C editor(s). Supplementary Guidance for Inclusion of Qualitative Research in Cochrane Systematic Reviews of Interventions. Cochrane Collaboration Qualitative Methods Group, 2011.

\section{Harden 2018}

Harden A. Thomas J, Cargo M, Harris J, Pantoja T, Flemming K, et al. Cochrane Qualitative and Implementation Methods Group Guidance Series-paper 5: methods for integrating qualitative and implementation evidence within intervention effectiveness reviews. Journal of Clinical Epidemiology 2018;97:70-8.

\section{Hayton 2013}

Hayton C, Clark A, Olive S, Browne P, Galey P, Knights E, et al. Barriers to pulmonary rehabilitation: characteristics that predict patient attendance and adherence. Respiratory Medicine 2013;107:401-7.

\section{Higgins 2011}

Higgins JPT, Green S (editors). Cochrane Handbook for Systematic Reviews of Interventions Version 5.1.0 (updated March 2011). The Cochrane Collaboration, 2011. Available from handbook.cochrane.org.

\section{Hillas 2015}

Hillas G, Perlikos F, Tsiligianni I, Tzanakis N. Managing co-morbidities in COPD. International Journal of Chronic Obstructive Pulmonary Disease 2015;10:95-109.

\section{Holland 2016}

Holland AE, Harrison SL, Brooks D. Multimorbidity, frailty and chronic obstructive pulmonary disease. Are the challenges for pulmonary rehabilitation in the name? . Chronic Respiratory Disease 2016;13(4):372-82. DOI: 10.1177\%2F1479972316670104

\section{Hong 2018}

Hong QN, Pluye P, Fàbregues $S$, Bartlett G, Boardman F, Cargo M, et al. Mixed methods appraisal tool (MMAT) version 2018. mixedmethodsappraisaltoolpublic.pbworks.com/w/file/ fetch/127916259/MMAT_2018_criteria-manual_2018-0801_ENG.pdf 2018.

\section{Ioannidis 2008}

Ioannidis JPA, Patsopoulos NA, Rothstein HR. Reasons or excuses for avoiding meta-analysis in forest plots. BMJ 2008;336(7658):1413-15. DOI: 10.1136/bmj.a117

\section{Keating 2011}

Keating A, Lee A, Holland AE. What prevents people with chronic obstructive pulmonary disease from attending pulmonary rehabilitation? A systematic review. Chronic Respiratory Disease 2011;8(2):88-9.

\section{Lefebvre 2009}

Lefebvre C, Manheimer E, Glanville J. Chapter 6: Searching for studies. In: Higgins JP, Green S, editor(s). Cochrane Handbook for Systematic Reviews of Interventions Version 5.1.0 (updated March 2011). The Cochrane Collaboration, 2011. Available from handbook.cochrane.org.

\section{Lewin 2015}

Lewin S, Glenton C, Munthe-Kaas H, Carleson B, Colvin C, Gülmezoglu M, et al. Using qualitative evidence in decision making for health and social interventions: an approach to assess confidence in findings from qualitative evidence syntheses (GRADE-CERQual). PLOS Medicine 2015;12(10):e1001895.

\section{Mannino 2008}

Mannino DM, Thorn D, Swensen A, Holguin F. Prevalence and outcomes of diabetes, hypertension and cardiovascular disease in COPD. European Respiratory Journal 2008;32(4): 962-9.

\section{Marshall 2018}

Marshall IJ, Noel-Storr AH, Kuiper J, Thomas J, Wallace BC. Machine learning for identifying randomised controlled trials: an evaluation and practitioner's guide. Research Synthesis Methods 2018;9(4):602-14. [PUBMED: 29314757]

McCarthy 2015

McCarthy B, Casey D, Devane D, Murphy K, Murphy E, Lacasse Y. Pulmonary rehabilitation for chronic obstructive pulmonary disease. Cochrane Database of Systematic Reviews 2015, Issue 2. DOI: 10.1002/14651858.CD003793.pub3

\section{McGowan 2016}

McGowan J, Sampson M, Salzwedel DM, Cogo E, Foerster V, Lefebvre C. PRESS peer review of electronic search strategies: 2015 guideline statement. Journal of Clinical Epidemiology 2016;75:40-6. [PUBMED: 27005575]

\section{McNamara 2013}

McNamara RJ, McKeough ZJ, McKenzie DK, Alison JA. Water-based exercise in COPD with physical co morbidities: a randomised controlled trial. European Respiratory Journal 2013;41(6):1284-91. DOI: $10.1183 / 09031936.00034312$

\section{Moher 2009}

Moher D, Liberati A, Tetzlaff J, Altman D. Preferred reporting items for systematic reviews and meta-analyses: the PRISMA statement. PLoS Medicine 2009;6(7): e1000097. DOI: 10.1371/journal.pmed.1000097

\section{Mourad 2016}

Mourad O, Hossam H, Fedorowicz Z, Elmagarmid A. Rayyan-a web and mobile app for systematic reviewers.

Targeted interventions and approaches to care for people living with chronic obstructive pulmonary disease and at least one other longterm condition: a mixed methods review (Protocol)

Copyright $\odot 2019$ The Cochrane Collaboration. Published by John Wiley \& Sons, Ltd. 
Systematic Reviews 2016;5(1):210. DOI: 10.1186/ s13643-016-0384-4

\section{Muth 2018}

Muth C, Blom JW, Smith SM, Johnell K, GonzalezGonzalez I, Nguyen TS, et al. Evidence supporting the best clinical management of patients with multi morbidity and polypharmacy: a systematic guideline review and expert consensus. Journal of Internal Medicine 2018 Oct 24 Epub ahead of print]. DOI: 10.1111/joim. 12842

NHS England 2018

NHS England. Right Care scenario: the variation between sub-optimal and optimal pathways. www.england.nhs.uk/ rightcare/wp-content/uploads/sites/40/2018/02/clarasstory-multimorbidity-full-narrative.pdf 2018.

\section{NICE 2018}

National Guideline Centre. Multimorbidity: clinical assessment and management. www.nice.org.uk/guidance/ ng56 (accessed prior to 23 January 2019).

\section{Noel-Storr 2018}

Noel-Storr AH, Project Transform team. Cochrane Crowd: new ways of working together to produce health evidence. Evidence Live; 2018 June 18-20; Oxford. 2018.

\section{Pluye 2011}

Pluye P, Robert E, Cargo M, Bartlett G, O'Cathain, Griffiths F, et al. Proposal: a mixed methods appraisal tool for systematic mixed studies reviews. mixedmethodsappraisaltoolpublic.pbworks.com. Archived by WebCite ${ }^{\circledR}$ at www.webcitation.org/5tTRTc9yJ 2011.

\section{Pollok 2018}

Pollok J, van Agteren JEM, Carson-Chahhoud KV. Pharmacological interventions for the treatment of depression in chronic obstructive pulmonary disease. Cochrane Database of Systematic Reviews 2018, Issue 12. DOI: 10.1002/14651858.CD012346.pub2

\section{Pollok 2019}

Pollok J, van Agteren JEM, Esterman AJ, Carson-Chahhoud KV. Psychological therapies for the treatment of depression in chronic obstructive pulmonary disease. Cochrane Database of Systematic Reviews 2019, Issue 3. DOI: 10.1002/14651858.CD012347

\section{Raherison 2018}

Raherison C, Ouaalaya E, Bernady A, Casteigt J, NocentEijnani C, Falque L, et al. Comorbidities and COPD severity in a clinical-based cohort. BMC Pulmonary Medicine 2018;18:117.

Review Manager 2014 [Computer program]

The Cochrane Collaboration. Review Manager 5 (RevMan 5). Version 5.3. Copenhagen: Nordic Cochrane Centre, The Cochrane Collaboration, 2014.

\section{Rijken 2016}

Rijken M, Struckmann V, van der Heide, Hujala A, Barbabella F, van Ginneken E, et al. How to improve care for people with multi morbidity in Europe?. www.euro.who.int/__data/assets/pdf_file/0004/337585/
PB_23.pdf?ua $=1$ (accessed prior to 23 January 2019). [ISSN 1997-8073]

Rijken 2018

Rijken M, Hujala A, van Ginneken E, Melchiorre MG, Groenewegen P, Schellevis F. Managing multi morbidity: profiles of integrated care approaches targeting people with multiple chronic conditions in Europe. Health Policy 2018; 122(1):44-52. DOI: 10.1016/j.healthpol.2017.10.002.

\section{Salisbury 2018}

Salisbury C. Management of multi morbidity using a patient-centred care model: a pragmatic cluster-randomised trial of the 3D approach. Lancet 2018;392(10141):41-50. DOI: $10.1016 / S 0140-6736(18) 31308-4$

\section{Sinnot 2013}

Sinnot C, McHugh S, Browne J, Bradley C. GPs perspectives on the management of patients with multi morbidity: systematic review and synthesis of qualitative research. $B M J$ Open 2013;3:e003610.

\section{Smith 2014}

Smith MC, Wrobel JP. Epidemiology and clinical impact of major co morbidities in patients with COPD. International Journal of Chronic Obstructive Pulmonary Disease 2014;9: $871-88$.

\section{Smith 2016}

Smith SM, Wallace E, O'Dowd T, Fortin M. Interventions for improving outcomes in patients with multimorbidity in primary care and community settings. Cochrane Database of Systematic Reviews 2016, Issue 3. DOI: 10.1002/ 14651858.CD006560.pub3

Tariq 2013

Tariq S, Woodman J. Using mixed methods in health research. Journal of the Royal Society of Medicine Short Reports 2013;4(6):2042533313479197. DOI: 10.1177/ 2042533313479197

\section{Thomas 2008}

Thomas J, Harden A. Methods for the thematic synthesis of qualitative research in systematic reviews. Biomed Central Medical Research Methodology 2008;8:45. DOI: 10.1186/ 1471-2288-8-45

United Nations 2013

United Nations, Department of Economic and Social Affairs, Population Division. World population ageing. www.un.org/en/development/desa/population/ publications/pdf/ageing/WorldPopulationAgeing2013.pdf. [accessed May 2019) 2013:1-114.

\section{Usmani 2011}

Usmani ZA, Carson KV, Cheng JN, Esterman AJ, Smith BJ. Pharmacological interventions for the treatment of anxiety disorders in chronic obstructive pulmonary disease. Cochrane Database of Systematic Reviews 2011, Issue 11. DOI: 10.1002/14651858.CD008483.pub2

\section{Usmani 2017}

Usmani ZA, Carson KV, Heslop K, Esterman AJ, De Soyza A, Smith BJ. Psychological therapies for the treatment of anxiety disorders in chronic obstructive pulmonary disease.

Targeted interventions and approaches to care for people living with chronic obstructive pulmonary disease and at least one other long- 
Cochrane Database of Systematic Reviews 2017, Issue 3.

DOI: 10.1002/14651858.CD010673.pub2

\section{Walsh 2013}

Walsh JR, McKeough ZJ, Morris NR, Chang AT, Yerkovich ST, Seale HE, et al. Metabolic disease and participant age are independent predictors of response to pulmonary rehabilitation. Journal of Cardiopulmonary Rehabilitation and Prevention 2013;33:249.

\section{World Health Organisation 2018}

World Health Organisation. Chronic obstructive pulmonary disease (COPD). www.who.int/respiratory/ copd/en/ 2018; Vol. (accessed 1 August 2018).

\section{Wyatt 2014}

Wyatt KD, Stuart LM, Brito JP, Carranza LB, Domecq JP, Prutsky GJ, et al. Out of context: clinical practice guidelines and patients with multiple chronic conditions: a systematic review. Medical Care 2014;52(3):S92-100.

* Indicates the major publication for the study

\section{ADDITIONAL TABLES}

Table 1. Framework and map of interventions identified from included studies

\begin{tabular}{l|l|l|}
\hline $\begin{array}{l}\text { Interven- } \\
\text { tions (identified from GOLD } \\
\begin{array}{l}2019 \text { guideline and Cochrane } \\
\text { Airways subtopic list.) }\end{array}\end{array}$ & $\begin{array}{l}\text { Study } \\
\text { mixed methods) }\end{array}$ \\
\hline $\begin{array}{l}\text { Reducing risk factors } \\
\circ \text { Smoking cessation }\end{array}$ & \\
\hline $\begin{array}{l}\text { Vaccination } \\
\circ \text { Pneumococcal } \\
\circ \text { Influenza }\end{array}$ & \\
\hline
\end{tabular}

- Pharmacotherapies

○ Short-acting inhalers (e.g. SABA)

- Long-acting inhalers

(e.g. LABA, LAMA, ICS)

- Phosphodiesterase-4

(PDE4) inhibitors (e.g.

Roflumilast)

- Mucolytic agents

- Combination

inhalers and triple therapy

- Methyl xanthines

- Oral corticosteroids

- Antibiotics

- Statins

- Alpha-1 antitrypsin

augmentation therapy

- Biomarker mediated

therapy

- Rehabilitation, education

and self-management

o Pulmonary

rehabilitation

Targeted interventions and approaches to care for people living with chronic obstructive pulmonary disease and at least one other long- 
Table 1. Framework and map of interventions identified from included studies (Continued)

$\circ$ Exercise therapy (e.

g. upper limb exercise, ongoing physical exercise after

pulmonary rehabilitation)

$\circ$ Complementary

therapies (e.g. active mind-

body therapy, Tai chi, singing)

- Education and self-

management

- Organisation of care

- Support services (e.

g. social care, specialist

respiratory nurse)

- Integrated care

- Telehealthcare

- Digital management

interventions

- Home care

- Integrated disease

management (e.g. disease

management programming)

- Interventions to

promote or increase adherence

to PR or other treatments

- Other treatments

- Oxygen therapy and

ventilatory support (e.g. NIV, ambulatory oxygen)

- Nutritional support

- Lung volume

reduction surgery

○ Lung

transplantation

- Supportive,

palliative, end of life and

hospice care

- Psychotherapy

o interventions for

sexual dysfunction

○ Self-help groups

Targeted interventions and approaches to care for people living with chronic obstructive pulmonary disease and at least one other long- 


\section{A P P E N D I C E S}

\section{Appendix I. Definitions of study designs used in this review}

Randomised study design

Observational,non-randomised study designs

\section{Appendix 2. MEDLINE (Ovid) search strategy}

\section{Search to identify reports of RCTs}

1 Lung Diseases, Obstructive/

2 exp Pulmonary Disease, Chronic Obstructive/

3 emphysema\$.tw.

4 (chronic $\$$ adj3 bronchiti\$).tw.

5 (obstruct $\$$ adj3 (pulmonary or lung $\$$ or airway $\$$ or airflow $\$$ or bronch $\$$ or respirat $\$$ )).tw.

6 (COPD or COAD or COBD or AECB or AECOPD).ti,ab.

7 or/1-6

8 exp COMORBIDITY/

9 (comorbid\$ or co-morbid\$).tw.

10 (multidisease\$ or multi-disease $\$$ or ((multiple or coexist\$ or co-exist\$) adj2 (illness\$ or disease $\$$ or condition\$ or syndrom\$ or disorder\$))).ti,ab.

11 (multimorbid\$ or multi-morbid\$).tw.

12 exp Chronic Disease/

13 (chronic\$ adj2 (illness\$ or disease\$ or condition\$ or disorder\$)).tw.

14 other health condition\$.tw.

15 other medical condition\$.tw.

16 (associated adj2 (disease\$ or disorder\$ or condition $\$$ or illness\$ or syndrome\$)).tw.

17 or/8-16

187 and 17

19 (controlled clinical trial or randomized controlled trial).pt.

20 (randomized or randomised).ab,ti.

21 placebo.ab,ti.

22 dt.fs.

23 randomly.ab,ti.

24 trial.ab,ti.

25 groups.ab,ti.

26 or/ $19-25$

27 Animals/

28 Humans/

2927 not (27 and 28)

3026 not 29

3118 and 30

\section{Search to identify reports of qualitative studies}

1 Lung Diseases, Obstructive/

2 exp Pulmonary Disease, Chronic Obstructive/

3 emphysema\$.tw.

4 (chronic $\$$ adj3 bronchiti\$).tw.

5 (obstruct $\$$ adj3 (pulmonary or lung $\$$ or airway $\$$ or airflow $\$$ or bronch $\$$ or respirat $\$$ )).tw.

Targeted interventions and approaches to care for people living with chronic obstructive pulmonary disease and at least one other longterm condition: a mixed methods review (Protocol)

Copyright $@ 2019$ The Cochrane Collaboration. Published by John Wiley \& Sons, Ltd. 
6 (COPD or COAD or COBD or AECB or AECOPD).ti,ab.

7 or/1-6

8 exp COMORBIDITY/

9 (comorbid\$ or co-morbid\$).tw.

10 (multidisease $\$$ or multi-disease $\$$ or ( multiple or coexist $\$$ or co-exist $\$$ ) adj2 (illness $\$$ or disease $\$$ or condition $\$$ or syndrom $\$$ or disorder\$))).ti,ab.

11 (multimorbid\$ or multi-morbid\$).tw.

12 exp Chronic Disease/

13 (chronic $\$$ adj2 (illness\$ or disease $\$$ or condition $\$$ or disorder\$)).tw.

14 other health condition $\$$.tw.

15 other medical condition $\$$.tw.

16 (associated adj2 (disease $\$$ or disorder $\$$ or condition $\$$ or illness $\$$ or syndrome $\$$ )).tw.

17 or/8-16

187 and 17

19 qualitative research/

20 Interview/

21 (theme\$ or thematic).mp.

22 qualitative.af.

23 Nursing Methodology Research/

24 questionnaire\$.mp.

25 ethnological research.mp.

26 ethnograph\$.mp.

27 ethnonursing.af.

28 phenomenol\$.af.

29 (grounded adj (theor\$ or study or studies or research or analys?s)). af.

30 (emic or etic or hermeneutic\$ or heuristic\$ or semiotic\$).af. or (data adj1 saturat\$).tw. or participant observ\$.tw.

31 (social construct $\$$ or (postmodern\$ or post-structural\$) or (post structural $\$$ or poststructural\$) or post modern\$ or post-modern\$ or feminis\$ or interpret\$).mp.

32 (action research or cooperative inquir\$ or co operative inquir\$ or co-operative inquir\$).mp.

33 (humanistic or existential or experiential or paradigm\$).mp.

34 (field adj (study or studies or research)).tw.

35 human science.tw.

36 biographical method.tw.

37 theoretical sampl\$.af.

38 ((purpos\$ adj4 sampl\$) or (focus adj group\$)).af.

39 (account or accounts or unstructured or openended or open ended or text\$ or narrative\$).mp.

40 (life world or life-world or conversation analys?s or personal experience $\$$ or theoretical saturation).mp.

41 ((lived or life) adj experience\$).mp.

42 cluster sampl\$.mp.

43 observational method\$.af.

44 content analysis.af.

45 (constant adj (comparative or comparison)).af.

46 ((discourse\$ or discurs\$) adj3 analys?s).tw.

47 narrative analys?s.af.

48 or/ $19-47$

4918 and 48

Targeted interventions and approaches to care for people living with chronic obstructive pulmonary disease and at least one other long- 
WHAT'S NEW

\begin{tabular}{|c|c|c|}
\hline Date & Event & Description \\
\hline 14 August 2019 & Amended & $\begin{array}{l}\text { Two references corrected to reflect that they are Cochrane Reviews rather than protocols (Pollok 2018; } \\
\text { Pollok 2019). }\end{array}$ \\
\hline
\end{tabular}

\section{CONTRIBUTIONS OFAUTHORS}

SJ: drafting of Background and Methods sections of the protocol, screening references, study selection, data extraction, risk of bias assessment, data analysis, GRADE assessment, write-up of full review.

MM: drafting of Background and Methods sections of the protocol, conceptual and clinical advice on protocol, analysis and interpretation, approval of final draft of full review.

ED: drafting of Background and Methods sections of the protocol, screening references, study selection, data extraction, risk of bias assessment, data analysis, GRADE assessment, write-up of full review.

$\mathrm{SH}$ : drafting of Background and Methods sections of the protocol, arbitrating conflicts, analysis and interpretation, approval of final draft of full review.

ES: design and conduct of search strategies, drafting of 'Search methods' section, database search of references, analysis and interpretation, approval of final draft of full review, arranging peer review of search strategy.

$\mathrm{AH}$ : drafting of Background and Methods sections of the protocol, conceptual and clinical advice on protocol, analysis and interpretation, approval of final draft of full review.

\section{Contributions of editorial team}

Rebecca Fortescue (Coordinating Editor): edited the protocol; advised on methodology; approved the protocol prior to publication. Chris Cates (Coordinating Editor): checked the planned methods.

Sally Spencer: edited the protocol; advised on content and review concept.

Emma Jackson (Assistant Managing Editor): coordinated the editorial process, conducted peer review; edited the references.

\section{DECLARATIONS OF INTEREST}

SJ: is employed full-time as a systematic reviewer, paid by an NIHR programme grant to complete work on this review.

MM: is a clinician at the Department of Respiratory Medicine, Galway University Hospital, Ireland.

ED: is the managing editor of Cochrane Airways group, St George's, University of London. This review will be managed and sent out to peer review by Emma Jackson.

SH: is a reader at the School of Health and Social Care, Teesside University, United Kingdom.

ES: is an information specialist, employed by the NIHR core grant in the Cochrane Airways group, St George's, University of London. $\mathrm{AH}$ : is a professor of physiotherapy at the School of Allied Health, at La Trobe University, Australia.

Targeted interventions and approaches to care for people living with chronic obstructive pulmonary disease and at least one other long- 18 term condition: a mixed methods review (Protocol)

Copyright $\odot 2019$ The Cochrane Collaboration. Published by John Wiley \& Sons, Ltd. 


\section{SOURCES OF SUPPORT}

\section{Internal sources}

- National Institute for Health Research, UK

Cochrane Programme Grant 16/114/21: NHS priorities in the management of chronic respiratory disease

\section{External sources}

- The authors declare that no such funding was received for this systematic review, Other. 